\title{
Convolution, filtering, and multiplexing in fractional Fourier domains and their relation to chirp and wavelet transforms
}

\author{
Haldun M. Ozaktas and Billur Barshan \\ Department of Electrical Engineering, Bilkent University, 06533 Bilkent, Ankara, Turkey \\ David Mendlovic
}

Faculty of Engineering, Tel Aviv University, 69978 Tel Aviv, Israel

Levent Onural

Department of Electrical Engineering, Bilkent University, 06533 Bilkent, Ankara, Turkey

Received June 1, 1993; accepted August 18, 1993

\begin{abstract}
A concise introduction to the concept of fractional Fourier transforms is followed by a discussion of their relation to chirp and wavelet transforms. The notion of fractional Fourier domains is developed in conjunction with the Wigner distribution of a signal. Convolution, filtering, and multiplexing of signals in fractional domains are discussed, revealing that under certain conditions one can improve on the special cases of these operations in the conventional space and frequency domains. Because of the ease of performing the fractional Fourier transform optically, these operations are relevant for optical information processing.
\end{abstract}

\section{INTRODUCTION}

Whenever we are confronted with an operator, it is natural to inquire into the effect of repeated applications of that operator, which might be considered as its integer powers. A further extension is to inquire what meaning may be attached to fractional powers of that operator. Examples of this are common. ${ }^{1-3}$

The fractional Fourier transform was defined mathematically by McBride and Kerr, ${ }^{4}$ based on research by Namias. ${ }^{5}$ In Refs. $1-3$ and 6 we defined the fractional Fourier-transform operator based on physical considerations, discovering that our definition is equivalent to that given in Ref. 4. In this paper we show how the twodimensional fractional Fourier transform can be realized optically and discuss various mathematical and physical properties.

An alternative definition of the fractional Fourier transform was suggested by Lohmann ${ }^{7}$ and later shown to be equivalent to the original definition. ${ }^{8}$ In retrospect this alternative definition is seen as one of the most important properties of the fractional transform.

In this paper we begin in Section 2 by distilling the essential concepts, definitions, and results of previous research. A number of miscellaneous novel results are derived in the process. In Section 3 we then discuss the relationship of fractional Fourier transforms to chirp transforms. This provides the basis of the concept of fractional domains, which are generalizations of the conventional space and frequency domains. Next, in Section 4, we discuss the relationship to wavelet transforms. Then we move on to convolution and compaction in fractional domains (Sections 5 and 6). Based on the material devel- oped in these sections, we then discuss filtering in fractional domains, showing that under certain circumstances noise separation can be realized effectively in fractional Fourier domains (Sections 7 and 8). Finally we discuss the concept of multiplexing in fractional domains, showing that, for certain signal Wigner distributions, efficient multiplexing can be realized in fractional domains (Section 9).

In most of this paper we work with continuous signals that are represented as functions of space or spatial frequency. Temporal interpretations of our discussions can be provided easily to those interested in them. Discrete signals are discussed only briefly, leaving further development to future research.

\section{PRELIMINARIES}

A. Notation and Definitions

The two functions $f$ and $F$ are a Fourier pair if

$$
\begin{aligned}
& F(\nu)=\int_{-\infty}^{\infty} f(x) \exp (-2 \pi i \nu x) \mathrm{d} x, \\
& f(x)=\int_{-\infty}^{\infty} F(\nu) \exp (2 \pi i \nu x) \mathrm{d} \nu .
\end{aligned}
$$

In operator notation we write $F=\mathscr{F}_{\mathcal{F}} f$. It is a fact that $\mathscr{F}^{2} f(x)=f(-x)$ and that $\mathscr{F}^{4} f(x)=f(x)$. Here, $\mathscr{F}^{j}$ means that the operator $\mathscr{F}$ is applied $j$ times in succession.

Consider the equation

$$
f^{\prime \prime}(x)+4 \pi^{2}\left[(2 n+1) / 2 \pi-x^{2}\right] f(x)=0 .
$$

By taking the Fourier transform of Eq. (3) and using elementary identities regarding the transforms of deriva- 
tives and moments, we can show that

$$
F^{\prime \prime}(\nu)+4 \pi^{2}\left[(2 n+1) / 2 \pi-\nu^{2}\right] F(\nu)=0,
$$

where $F(\nu)=\mathscr{F}[f(x)]$. Because Eq. (4) is identical to Eq. (3) in form, it is easy to accept the well-known fact that solutions of this equation, known as Hermite-Gauss functions, are eigenfunctions of the Fourier transform operation. ${ }^{9,10}$ Normalized so that they form an orthonormal set, these functions are given by

$$
\tilde{\Psi}_{n}(x)=\frac{2^{1 / 4}}{\sqrt{2^{n} n !}} H_{n}(\sqrt{2 \pi} x) \exp \left(-\pi x^{2}\right)
$$

for $n=0,1,2, \ldots$ These functions satisfy the eigenvalue equation $^{9,10}$

$$
\mathscr{F}\left[\tilde{\Psi}_{n}(x)\right]=\lambda_{n} \tilde{\Psi}_{n}(x)
$$

with $\lambda_{n}=i^{-n}$ being the eigenvalue corresponding to the $n$th eigenfunction. (Additional comments on the solutions of this equation may be found in Appendix A.) Because the Hermite-Gaussian functions form a complete set, one can than calculate the Fourier transform of an arbitrary function by expressing it in terms of these eigenfunctions as follows:

$$
\begin{aligned}
f(x) & =\sum_{n=0}^{\infty} \tilde{A}_{n} \tilde{\Psi}_{n}(x), \\
\tilde{A}_{n} & =\int_{-\infty}^{\infty} \tilde{\Psi}_{n}(x) f(x) \mathrm{d} x, \\
\mathscr{F}[f(x)] & =\sum_{n=0}^{\infty} \tilde{A}_{n} i^{-n} \tilde{\Psi}_{n}(x) .
\end{aligned}
$$

The fractional Fourier transform operator of order $a$ may be defined through its effect on the eigenfunctions of the conventional Fourier operator,

$$
\mathscr{F}^{a}\left[\tilde{\Psi}_{n}(x)\right]=\lambda_{n}{ }^{a} \tilde{\Psi}_{n}(x)=i^{-a n} \tilde{\Psi}_{n}(x) ;
$$

that is, the fractional operator is defined to have the same eigenfunctions as the original operator, and the eigenvalues $\lambda_{n}{ }^{a}$. If we define our operator to be linear, the fractional transform of an arbitrary function can be expressed as

$$
\left\{\mathscr{F}^{a}[f(x)]\right\}(x)=\sum_{n=0}^{\infty} \tilde{A}_{n} i^{-a n} \tilde{\Psi}_{n}(x) .
$$

This definition is identical to that given in Refs. 1 and 2 if we substitute 1 for the value of the physical scale parameter $s$ (with dimension of length) that appear in those papers. (Details may be found in Appendix B.)

The definition can also be cast in the form of a general linear transformation with kernel $B_{a}\left(x, x^{\prime}\right)$ by insertion of Eq. (8) into Eq. (11) (Ref. 3):

$$
\begin{aligned}
\left\{\mathscr{F}^{a}[f(x)]\right\}(x)= & \int_{-\infty}^{\infty} B_{a}\left(x, x^{\prime}\right) f\left(x^{\prime}\right) \mathrm{d} x^{\prime}, \\
B_{a}\left(x, x^{\prime}\right)= & \sum_{n=0}^{\infty} \lambda_{n}{ }^{a} \tilde{\Psi}_{n}(x) \tilde{\Psi}_{n}\left(x^{\prime}\right) \\
= & 2^{1 / 2} \exp \left[-\pi\left(x^{2}+x^{\prime 2}\right)\right] \\
& \times \sum_{n=0}^{\infty} \frac{i^{-a n}}{2^{n} n !} H_{n}(\sqrt{2 \pi} x) H_{n}\left(\sqrt{2 \pi} x^{\prime}\right) .
\end{aligned}
$$

A simpler form is given for $0<|\phi|<\pi$ (i.e., $0<|a|<2$ ) (Refs. 4 and 7):

$$
\begin{aligned}
B_{a}\left(x, x^{\prime}\right)= & \frac{\exp [-i(\pi \hat{\phi} / 4-\phi / 2)]}{|\sin \phi|^{1 / 2}} \\
& \times \exp \left[i \pi\left(x^{2} \cot \phi-2 x x^{\prime} \csc \phi+x^{\prime 2} \cot \phi\right)\right],
\end{aligned}
$$

where $\phi=a \pi / 2$ and $\hat{\phi}=\operatorname{sgn}(\sin \phi)$. The kernel is defined separately for $a=0$ and $a=2$ as $B_{0}\left(x, x^{\prime}\right)=$ $\delta\left(x-x^{\prime}\right)$ and $B_{2}\left(x, x^{\prime}\right)=\delta\left(x+x^{\prime}\right)$, respectively.

The above constitutes the definition of the fractional Fourier transform in its purest mathematical form, purged of the physical constants appearing in Refs. 2 and 3. Some essential properties are listed below; others may be found in Refs. 1-3:

1. The fractional Fourier transform operator is linear.

2. The first-order transform $\mathscr{F}^{1}$ corresponds to the conventional Fourier transform $\mathscr{F}^{\circ}$.

3. The fractional operator is additive, $\mathscr{F}^{a_{1}} \mathscr{F}^{a_{2}}=\mathscr{F}^{a_{1}+a_{2}}$.

When there is a possibility of confusion, the coordinate variable in the $a$ th fractional Fourier domain is denoted as $x_{a}$, so that $x_{0}=x$ and $x_{1}=\nu$, and the functional form of the signal in the ath domain is denoted as $f_{a}$, so that $f_{0}=f$ and $f_{1}=F$. In this paper all $x_{a}$ are considered dimensionless.

We also note that, although one-dimensional signals are considered throughout this paper for notational simplicity, straightforward generalization of the results to two dimensions is possible.

The fractional Fourier transform is of great interest from an optics perspective because it describes propagation in quadratic graded index (GRIN) media. If a light distribution of the form $f(x, y)$ is incident upon one end of a piece of such a medium of length $a L$, at the other end we observe its ath two-dimensional fractional Fourier transform. Thus a piece of such a medium can be used for analog computation of the fractional Fourier transform. This is discussed in detail in Refs. 1-3 and briefly in Appendix B.

\section{B. Relation to Wigner Distributions}

Now we discuss one of the most important properties of the fractional Fourier transform. In fact it has been proposed as an alternative definition of the fractional Fourier transform ${ }^{7}$ and later proved to be equivalent to the definition given above. ${ }^{8}$ An alternative and shorter proof of this equivalence is given in Appendix $\mathbf{C}$.

This property states that performing the ath fractional Fourier transform operation corresponds to rotating the Wigner distribution by an angle $\phi=a(\pi / 2)$ in the clockwise direction. The Wigner distribution of a function is defined as

$$
\begin{aligned}
W[f(x)] & =W(x, \nu) \\
& =\int f\left(x+x^{\prime} / 2\right) f^{*}\left(x-x^{\prime} / 2\right) \exp \left(-2 \pi i \nu x^{\prime}\right) \mathrm{d} x^{\prime}
\end{aligned}
$$

$W(x, \nu)$ can also be expressed as a function of $F(\nu)$, or indeed as a function of any fractional transform of $f(x)$. 
Further discussion of the Wigner distribution may be found in Refs. 11-13. Here we mention some properties that are most relevant:

1. $|f(x)|^{2}=\int W(x, \nu) \mathrm{d} \nu$.

2. $|F(\nu)|^{2}=\int W(x, \nu) \mathrm{d} x$.

3. The total energy is $\int W(x, \nu) \mathrm{d} x \mathrm{~d} \nu$.

4. Roughly speaking, $W(x, \nu)$ can be interpreted as a function that indicates the distribution of the signal energy over space and frequency. For a more precise discussion, see Refs. 11 and 12.

Let us also define the rotation operator $\mathbf{R}_{\phi}$ for twodimensional functions, corresponding to a counterclockwise rotation by $\phi$. Then, the property stated immediately before Eq. (15) can be expressed as

$$
\mathcal{W}\left[f_{a}\right]=\mathbf{R}_{-\phi} \mathcal{W}\left[f_{0}\right] .
$$

Because both the fractional Fourier transform and rotation operators are additive with respect to their parameters, this easily generalizes to

$$
\mathscr{W}\left[f_{a_{2}}\right]=\mathbf{R}_{\left(-\phi_{2}+\phi_{1}\right)} \mathcal{W}\left[f_{a_{1}}\right] .
$$

This relationship is relevant from an optics viewpoint. Wigner space is essentially a phase space and is analogous to a different kind of phase space defined in optics (with ray intercepts corresponding to the $x$ axis and ray angles corresponding to the $\nu$ axis). In Ref. 1 we show that the distribution of ray bundles in this phase space also rotates as a result of propagation through quadratic GRIN media.

An immediate corollary (indeed a restatement) of this property was first noticed by Lohmann and Soffer ${ }^{14}$ :

$$
\mathscr{R}_{\phi}[\mathcal{W}[f]]=\left|\mathscr{F}^{a}[f]\right|^{2},
$$

where the operator $\mathscr{R}_{\phi}$ is the Radon transform evaluated at the angle $\phi$. The Radon transform of a two-dimensional function is its projection on an axis making angle $\phi$ with the $x_{0}$ axis. It may be defined in terms of the rotation operator as

$$
\mathscr{R}_{\phi}\left[W\left(x_{0}, x_{1}\right)\right]=\int \mathbf{R}_{-\phi}\left[W\left(x_{0}, x_{1}\right)\right] \mathrm{d} x_{1} .
$$

Equation (18) is a generalization of the first two properties given above following Eq. (15).

Let us also define the slice operator $\mathscr{Y}_{\phi}$ such that $\varphi_{\phi} p\left(x_{0}, x_{1}\right)$ is a one-dimensional function that takes the values of $p\left(x_{0}, x_{1}\right)$ along a line making an angle $\phi$ with the $x_{0}$ axis. The projection-slice theorem states that ${ }^{15}$

$$
\mathscr{F}_{\mathscr{P}} \mathscr{R}_{\phi} W[f]=\mathscr{S}_{\phi} \mathscr{F}_{2 \mathrm{D}} \mathcal{W}[f],
$$

where $\mathscr{F}_{2 \mathrm{D}}$ is the two-dimensional Fourier transform operator. We also know ${ }^{11}$ that $\mathscr{F}_{2 \mathrm{D}} \mathcal{W}[f]=A(\mu,-y)$, where

$$
A(\mu, y)=\int f(x+y / 2) f^{*}(x-y / 2) \exp (-2 \pi i \mu x) \mathrm{d} x
$$

is the well-known ambiguity function. By combining this result and Eqs. (18) and (20), we obtain

$$
\mathscr{F}\left|\mathscr{F}^{a}[f]\right|^{2}=\mathscr{S}_{\phi}[A(\mu,-y)],
$$

which is the dual of Eq. (18). (It is easy to show that the special case $a=1$ is nothing but the result stating that the Fourier transform of the autocorrelation of a function is the absolute square of its Fourier transform.)

\section{Discrete Fractional Fourier Transforms}

At this point it is also appropriate to discuss the discrete fractional Fourier transform. Two functions are an $N$ point discrete Fourier transform pair if they satisfy

$$
\begin{aligned}
G_{l} & =\sum_{k=0}^{N-1} g_{k} w^{k l}, \\
g_{k} & =\sum_{l=0}^{N-1} G_{l} w^{-k l},
\end{aligned}
$$

where

$$
w^{k l}=\frac{1}{\sqrt{N}} \exp (-2 \pi i k l / N)
$$

In matrix form we write

$$
\begin{aligned}
& \mathbf{G}=\mathbf{F} \mathbf{g}, \\
& \mathbf{g}=\mathbf{F}^{-1} \mathbf{G},
\end{aligned}
$$

where $\mathbf{G}=\left[\mathrm{G}_{0}, G_{1}, \ldots, G_{N-1}\right]^{T}, \mathbf{g}=\left[g_{0}, g_{1}, \ldots, g_{N-1}\right]^{T}$, and $\mathbf{F}$ has $w^{(j-1)(k-1)}$ as its $(j, k)$ th element. It can be shown that $\mathbf{F}^{4}=\mathbf{1}$, with 1 denoting the identity matrix. A relevant identity is

$$
\sum_{l=0}^{N-1} w^{l j} w^{-l k}=\delta_{k j}
$$

which is nothing but a statement that the rows and columns of $\mathbf{F}$ constitute an orthonormal set of vectors. Thus $\mathbf{F}$ is a unitary matrix. ${ }^{16}$

Now consider the eigenvalue equation

$$
\mathbf{F}^{a} \mathbf{g}=\lambda \mathbf{g} \text {. }
$$

This has already been studied for $a=1$, and a method for obtaining orthogonal eigenvectors has been presented. ${ }^{16}$ This discussion is not repeated here. Because $\mathbf{F}^{4} \mathbf{g}=\mathbf{g}$, we again have $\lambda^{4}=1$, so that the eigenvalues are again given by

$$
\lambda_{n}=\exp (-i n \pi / 2)=i^{-n},
$$

with integer $n$.

We let $\mathbf{g}_{n}$ denote an eigenvector with eigenvalue $\lambda_{n}$, i.e.,

$$
\mathbf{F g}_{n}=\lambda_{n} \mathbf{g}_{n},
$$

and note for further reference that for integer $j$, repeated application of Eq. (31) gives

$$
\mathbf{F}^{j} \mathbf{g}_{n}=\lambda_{n}^{j} \mathbf{g}_{n} .
$$

Now let us consider the case of general $a$. The ath power of the matrix $\mathbf{F}$ can be expressed in terms of its integer powers ${ }^{16}$ :

$$
\begin{aligned}
\mathbf{F}^{a} & =\sum_{j=0}^{3} \mathbf{F}^{j} \alpha_{j}(a) \\
\alpha_{j}(a) & =\frac{1}{4} \sum_{k=1}^{4} \exp [i k(a-j) \pi / 2]
\end{aligned}
$$


for $0 \leq a \leq 1$. The operator $\mathbf{F}^{a}$ is unitary. ${ }^{16}$ From the same reference we also know that a fast fractional transform with serial time complexity $\sim N \log N$ exists.

In Appendix D we show that this way of defining the fractional discrete Fourier transform (by taking the fractional power of the discrete Fourier transform matrix) is fully analogous to our definition of the fractional Fourier transform [Eq. (10)]. That is, we show that the eigenfunctions of the fractional transform are the same as that of the original transform and that the eigenvalues are the corresponding power $a$ of the original eigenvalues. Thus, if Eq. (31) holds, we also have

$$
\mathbf{F}^{a} \mathbf{g}_{n}=\lambda_{n}{ }^{a} \mathbf{g}_{n}
$$

for $0 \leq a \leq 1$. By virtue of Eq. (32), this easily generalizes to all real values of $a$.

Because an orthonormal set of real eigenvectors can be found, ${ }^{16}$ any vector $g$ can be expressed in terms of these eigenvectors:

$$
\begin{aligned}
\mathbf{g} & =\sum_{n=1}^{N} A_{n} \mathbf{g}_{n}, \\
A_{n} & =\mathbf{g}_{n}{ }^{T} \mathbf{g},
\end{aligned}
$$

where $A_{n}$ denotes the expansion coefficient, which is found by taking the inner product of $\mathbf{g}_{n}$ and $\mathbf{g}$. The effect of the fractional operator can be expressed as

$$
\mathbf{F}^{a} \mathbf{g}=\sum_{n=1}^{N} A_{n} \lambda_{n}{ }^{a} \mathbf{g}_{n}
$$

by linearity and Eq. (35).

\section{RELATION TO CHIRP TRANSFORMS}

Great insight can be gained into the nature of chirp transforms by interpreting them in terms of fractional Fourier transforms. We begin by recalling the Wigner distribution of some elementary functions ${ }^{11}$ :

$$
\begin{aligned}
& f(x)=\exp \left(2 \pi i \nu_{c} x\right), \quad W(x, \nu)=\delta\left(\nu-\nu_{c}\right), \\
& f(x)=\delta\left(x-x_{c}\right), \quad W(x, \nu)=\delta\left(x-x_{c}\right), \\
& f(x)=\exp \left[2 \pi i\left(b_{2} x^{2} / 2+b_{1} x+b_{0}\right)\right] \text {, } \\
& W(x, \nu)=\delta\left(b_{2} x+b_{1}-\nu\right) .
\end{aligned}
$$

The first of these results says that the Wigner distribution of a pure harmonic is a line delta concentrated along $\nu=\nu_{0}$, parallel to the $x$ axis. The second says that the Wigner distribution of a delta function is a line delta concentrated along $x=x_{0}$, perpendicular to the $x$ axis. The third says that the Wigner distribution of a chirp function is a line delta making an angle $\phi=\tan ^{-1} b_{2}$ with the $x$ axis (Fig. 1).

Given the fact that the effect of fractional Fourier transforming is to rotate the Wigner distribution of a function, we suspect that a chirp function is the $a=0$ domain representation of pure harmonics or delta functions in other fractional Fourier domains.

First, we investigate the fractional Fourier transforms of the function $\delta\left(x_{0}-x_{0 c}\right)$, where $x_{0}=x$ and $x_{0 c}$ is a constant. This is done most conveniently by using the kernel given in Eq. (14):

$$
\begin{aligned}
f_{a}\left(x_{a}\right)= & \frac{\exp [-i(\pi \hat{\phi} / 4-\phi / 2)]}{|\sin \phi|^{1 / 2}} \\
& \times \exp \left[i \pi\left(x_{a}{ }^{2} \cot \phi-2 x_{a} x_{0 c} \csc \phi+x_{0 c}{ }^{2} \cot \phi\right)\right] .
\end{aligned}
$$

For $a=1(\phi=\pi / 2)$, this reduces to $\exp \left(-2 \pi i \nu x_{0 c}\right)$, with $\nu=x_{1}$, as expected. $f_{a}\left(x_{a}\right)$ should be considered an alternative representation of $f_{0}\left(x_{0}\right)$ in the ath fractional domain. In particular the $a=1$ st domain corresponds to the conventional Fourier domain. Because $f_{a}\left(x_{a}\right)$ is the ath transform of $f_{0}\left(x_{0}\right)$, its Wigner distribution must be a rotated version of that of $f_{0}\left(x_{0}\right)$. This is verified easily by writing the Wigner distribution of $f_{a}\left(x_{a}\right)$, now interpreted as a function in $x_{0}$ space, i.e., as $f_{a}\left(x_{0}\right)$ :

$$
\begin{aligned}
W\left(x_{0}, x_{1}\right) & =|\sin \phi|^{-1} \delta\left(x_{0} \cot \phi-x_{0 c} \csc \phi-x_{1}\right) \\
& =\delta\left(x_{0} \cos \phi-x_{0 c}-x_{1} \sin \phi\right),
\end{aligned}
$$

which we recognize as the Wigner distribution of $\delta\left(x_{0}-x_{0 c}\right)$, which is also $\delta\left(x_{0}-x_{0 c}\right)$, rotated by $-\phi$.

The above can be generalized easily. A delta function in the ath domain, $\delta\left(x_{a}-x_{0 c}\right)$, is in general a chirp function in the $a^{\prime}$ th domain. When $a^{\prime}=a+1$, in particular, it is a pure harmonic. The choice of the $x_{0}=x$ and $x_{1}=\nu$ axes is nothing but an arbitrary choice of the origin of the parameter $a$. The domain that we choose to designate as the $a=0$ th domain is associated with the $x_{0}=x$ axis, and the domain we choose to designate as the $a=1$ st domain is associated with the $x_{1}=\nu$ axis. In general, the ath domain is associated with the $x_{a}$ axis. Other than this, there is nothing special about any domain; what is called Wigner space has complete rotational symmetry. In other words we imagine that the Wigner distribution exists as a geometric entity, independently of the choice of coordinate axes. By introducing coordinate axes, we choose the origin of $a$. Depending on the Wigner distribution at hand, certain choices of $a$ may be more useful and simpler than others.

It is also instructive to consider the identity

$$
f(x)=f_{0}\left(x_{0}\right)=\int f_{0}\left(x^{\prime}\right) \delta\left(x_{0}-x^{\prime}\right) \mathrm{d} x^{\prime} .
$$

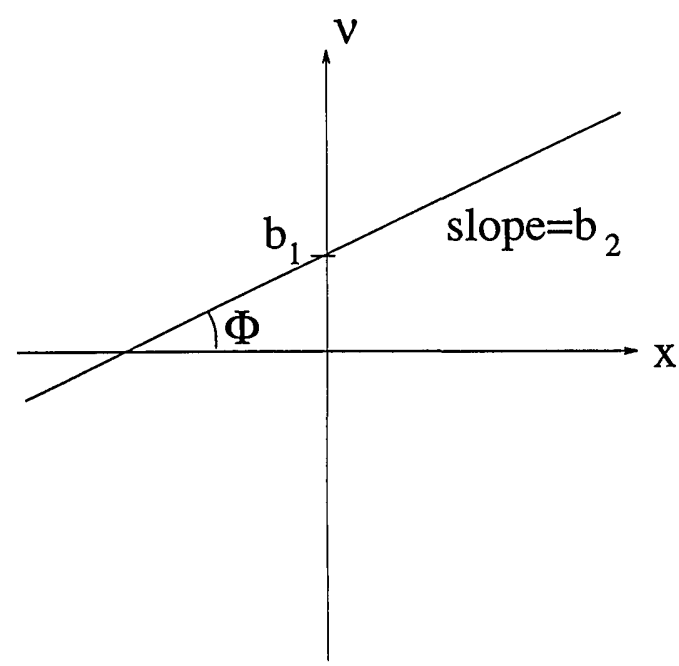

Fig. 1. Wigner distribution of a chirp function. 
Let us operate with $\mathscr{F}^{a}$ on both sides to obtain

$$
f_{a}\left(x_{a}\right)=\int f_{0}\left(x^{\prime}\right) \mathscr{F}^{a}\left[\delta\left(x_{0}-x^{\prime}\right)\right] \mathrm{d} x^{\prime} .
$$

We recognize $\mathscr{F}^{a}\left[\delta\left(x_{0}-x^{\prime}\right)\right]$, the chirp function given in Eq. (42), as the fractional Fourier transform kernel. [Special case: For $a=1$, the Fourier transform of $\delta\left(x_{0}-x^{\prime}\right)$ is the harmonic $\exp \left(-2 \pi i x_{1} x^{\prime}\right)=\exp \left(-2 \pi i \nu x^{\prime}\right)$, which is the kernel of the conventional Fourier transform.]

The above analysis can be easily repeated for harmonic functions of the form $\exp \left(2 \pi i \nu_{0 c} x_{0}\right)$, where $\nu_{0 c}$ is a constant. This reveals that fractional Fourier transforms of such functions are in general chirp functions, and their Wigner distributions are rotated versions of the original function.

The representation of a signal in the ath domain is simply what we call the ath fractional Fourier transform of the representation of the signal in the $a=0$ th (space) domain. If we know the representation of the signal in the $a^{\prime}$ th domain, we can find its representation in the ath domain by taking its $\left(a-a^{\prime}\right)$ th fractional transform. The representation of a (well-behaved) signal in the ath domain can be written as a superposition of delta functions in the same domain:

$$
f_{a}\left(x_{a}\right)=\int f_{a}\left(x^{\prime}\right) \delta\left(x_{a}-x^{\prime}\right) \mathrm{d} x^{\prime}
$$

or as a superposition of harmonics in the domain orthogonal to that domain [which is the $(a+1)$ th domain]:

$$
f_{a}\left(x_{a}\right)=\int F_{a}\left(\nu_{a}\right) \exp \left(2 \pi i \nu_{a} x_{a}\right) \mathrm{d} \nu_{a}
$$

(where $F_{a}=f_{a+1}, \nu_{a}=x_{a+1}$, and $\nu=\nu_{0}=x_{1}$ ), or in general as a superposition of chirp functions in some other $a^{\prime}$ th domain:

$$
f_{a}\left(x_{a}\right)=\int f_{a^{\prime}}\left(x_{a^{\prime}}\right) B_{a-a^{\prime}}\left(x_{a}, x_{a^{\prime}}\right) \mathrm{d} x_{a^{\prime}} .
$$

The kernel $B_{a-a^{\prime}}\left(x_{a}, x_{a^{\prime}}\right)$ is the $\left(a-a^{\prime}\right)$ th fractional Fourier transform kernel. Taking the $\Delta a$ th fractional Fourier transform of the representation of a signal in the $a^{\prime}$ th domain results in the representation of that signal in the $a=a^{\prime}+\Delta a$ th domain. This is equivalent to finding the projection of $f_{a^{\prime}}$ in the $a^{\prime}$ th domain onto the basis functions (which may be delta functions or harmonics) in the ath domain. The representation of these basis functions in the original $a^{\prime}$ th domain is chirp functions, so that the inner products by which the projections are found are in the form of chirp transforms. We summarize with the following:

1. Basis functions in the ath domain, whether they are delta functions or harmonics, are in general chirp functions in the $a^{\prime}$ th domain.

2. The representation of a signal in the ath domain can be obtained from the representation in the $a^{\prime}$ th domain by taking the inner product (projection) of the representation in the $a^{\prime}$ th domain with basis functions in the target $a$ th domain.

3. This operation, having the form of a chirp transform, is equivalent to taking the $\left(a-a^{\prime}\right)$ th fractional
Fourier transform of the representation in the $a^{\prime}$ th domain.

The chirp transform is of great relevance to optics. Setting temporal phenomena aside, we note that the chirp transform is important, if only because the Fresnel diffraction integral is of this form. Thus propagation in free space can be formulated as a chirp transform. Indeed, quadratic GRIN media are nothing but the limiting form of a homogeneous medium with positive lenses inserted at equal intervals to compensate for the diffraction spread. ${ }^{1}$ The resulting uniform properties of such media lead to the simplest relationship to fractional Fourier transforms. Nevertheless, propagation in free space can also be analyzed with a similar formalism. Furthermore, this leads us to an interpretation of fractional Fourier or chirp transforms as wavelet transforms, as we discuss in Section 4.

\section{RELATION TO WAVELET TRANSFORMS}

The fractional Fourier-transform kernels corresponding to different values of $a$ are closely related to a wavelet family. Using straightforward algebraic manipulations of Eq. (14) and making the change of variable $y=x_{a} \sec \phi$, we can write the transform of $f(x)$ as

$$
\begin{aligned}
g(y)= & f_{a}\left(\frac{y}{\sec \phi}\right)=C(\phi) \exp \left(-i \pi y^{2} \sin ^{2} \phi\right) \\
& \times \int \exp \left[i \pi\left(\frac{y-x^{\prime}}{\tan ^{1 / 2} \phi}\right)^{2}\right] f\left(x^{\prime}\right) \mathrm{d} x^{\prime} .
\end{aligned}
$$

Taking $\tan ^{1 / 2} \phi$ as the scale parameter, the convolution represented by the above integral is a wavelet transform in which the wavelet family is obtained from the quadratic phase function $w(x)=\exp \left(i \pi x^{2}\right)$ by scaling the coordinate and the amplitude by $\tan ^{1 / 2} \phi$ and $C(\phi)$, respectively.

It has recently been shown that the formulation of optical difffraction can be cast into a similar wavelet framework. ${ }^{17}$

In Section 7 we discuss the filtering of functions at different fractional Fourier domains. Thus, based on the above, these operations can also be interpreted as filtering at the corresponding wavelet transform domains.

\section{CONVOLUTION IN FRACTIONAL DOMAINS}

The convolution of the functions $f$ and $h$ in the $a$ th domain,

$$
g_{a}\left(x_{a}\right)=\mathscr{F}_{\mathfrak{F}} a[g]=\mathscr{F}^{a}[f] * \mathscr{F}^{a}[h]=f_{a}\left(x_{a}\right) * h_{a}\left(x_{a}\right),
$$

is denoted as $g=f * a$. When $a=0$, we have $g=f * h$, so that the binary operator $*$ is equivalent to the ordinary convolution operator. When $a=1$, we have $\mathscr{F}_{g}=$ $\mathscr{F} f * \mathscr{F} h$, which translates to $g=f h$, so that convolution in the $a=1$ st domain corresponds to ordinary multiplication. It is possible to show that convolution in the second and third domains are the same as that in the zeroth and first domains, respectively. Convolution in the $a=1 / 2$ th domain is something midway between convolution and multiplication of two functions.

Let us examine the effect of convolution on the Wigner distributions. First we recall the following properties of 
the Wigner distribution, which can be easily shown by use of the definition Eq. (15). ${ }^{12}$ If $g(x)=f(x) * h(x)$, then

$$
W_{g}(x, \nu)=\int W_{f}\left(x^{\prime}, \nu\right) W_{h}\left(x-x^{\prime}, \nu\right) \mathrm{d} x^{\prime} ;
$$

whereas, if $g(x)=f(x) h(x)$ (i.e., $\mathscr{F}[g]=\mathscr{F}[f] * \mathscr{F}[h])$, then

$$
W_{g}(x, \nu)=\int W_{f}\left(x, \nu^{\prime}\right) W_{h}\left(x, \nu-\nu^{\prime}\right) \mathrm{d} \nu^{\prime}
$$

The first of these results says that if two functions are convolved in the $x$ domain their Wigner distributions are convolved along the $x$ direction. The second says that if two functions are convolved in the $\nu$ domain their Wigner distributions are convolved along the $\nu$ direction. Next we consider the case in which the two functions are convolved in an arbitrary fractional domain. As a necessary consequence of the rotational symmetry of Wigner space (i.e., the arbitrariness of choice of origin of $a$ ), it follows that if two functions are convolved in the $x_{a}$ domain their Wigner distributions are convolved along the $x_{a}$ direction.

The multiplication, or product, of two functions $f$ and $h$ in the ath domain,

$$
g_{a}\left(x_{a}\right)=\mathscr{F}^{a}[g]=\mathscr{F}^{a}[f] \times \mathscr{F}^{a}[h]=f_{a}\left(x_{a}\right) h_{a}\left(x_{a}\right),
$$

is denoted as $g=f \stackrel{a}{x} h$. From this equation we can deduce that

$$
\begin{aligned}
& \mathscr{F}^{a+1}[f \stackrel{a}{\times} h]=\mathscr{F}^{a+1}[f] * \mathscr{F}^{a+1}[h]=\mathscr{F}^{a+1}\left[f^{a} *^{1} h\right], \\
& \mathscr{F}^{a-1}[f \stackrel{a}{\times} h]=\mathscr{F}^{a-1}[f] * \mathscr{F}^{a-1}[h]=\mathscr{F}^{a-1}\left[f^{a^{1}} h\right] .
\end{aligned}
$$

Likewise from Eq. (50) we can deduce that

$$
\begin{aligned}
& \mathscr{F}^{a+1}\left[f^{a} * h\right]=\mathscr{F}^{a+1}[f] \times \mathscr{F}^{a+1}[h]=\mathscr{F}^{a+1}\left[f^{a+1} \times{ }^{\circ} h\right], \\
& \mathscr{F}^{a-1}\left[f^{a} * h\right]=\mathscr{F}^{a-1}[f] \times \mathscr{F}^{a-1}[h]=\mathscr{F}^{a-1}\left[f^{a-1} \times\right] .
\end{aligned}
$$

These lead to the identities

$$
\begin{gathered}
f \stackrel{a}{\times} h=f^{a *^{1}} h=f^{a \bar{*}^{1}} h, \\
f^{a} h=f^{a+1} \stackrel{\times}{\times} h^{*}=f^{a-1} \check{\times}^{1} h .
\end{gathered}
$$

These results mean that convolution in the ath domain corresponds to multiplication in the $(a+1)$ th and $(a-1)$ th domains and that multiplication in the $a$ th domain corresponds to convolution in the $(a+1)$ th and $(a-1)$ th domains. From these results we can also see that convolution (multiplication) in the ath domain is the same as convolution (multiplication) in the $(a+2)$ th and $(a-2)$ th domains.

In a previous paper we defined fractional convolution differently. ${ }^{2}$ The $a_{p}$ th convolution of $f$ and $h$ was defined as

$$
\mathscr{F}^{a}\left[f^{a_{p}} h\right]=\mathscr{F}^{a}[f] \times \mathscr{F}^{a}[h] .
$$

According to this definition, the $a_{p}=1$ st convolution corresponds to ordinary convolution, so that we speak of the $a_{p}$ th convolution, whereas above we spoke of convolution in the ath domain. We see that what was defined as the $a_{p}$ th convolution previously corresponds to multiplication in the ath domain in this paper.

\section{COMPACTION IN FRACTIONAL DOMAINS}

A function is said to be compact in the ath domain if its value is zero outside an interval around the origin. Compaction in any domain can be realized by multiplying the function with the window

$$
\operatorname{rect}\left(\frac{x_{a}-x_{a c}}{\Delta x_{a}}\right)
$$

in that domain. That is, if $g_{a}\left(x_{a}\right)$ represents the compacted function,

$$
g_{a}\left(x_{a}\right)=\operatorname{rect}\left(\frac{x_{a}-x_{\alpha c}}{\Delta x_{a}}\right) f_{a}\left(x_{a}\right) .
$$

Compaction in the $a=1$ st domain is what is conventionally known as bandpass filtering. It serves as a component operation in many applications.

We now discuss the effect of compaction in a certain domain on the Wigner distribution. Because compaction involves multiplication with a rectangle function, this implies convolution of the Wigner distribution of $f_{a}\left(x_{a}\right)$ with the Wigner distribution of the rectangle function, in the direction orthogonal to $x_{a}$ (the direction of the $x_{a+1}$ axis). (See the discussion in Section 5.) Thus we first proceed to find the Wigner distribution of the rectangle function:

$$
\begin{aligned}
W_{\text {rect }}\left(x_{a}, \nu_{a}\right)= & \int \operatorname{rect}\left(\frac{x_{a}+x^{\prime} / 2-x_{a c}}{\Delta x_{a}}\right) \\
& \times \operatorname{rect}\left(\frac{x_{a}-x^{\prime} / 2-x_{a c}}{\Delta x_{a}}\right) \exp \left(-2 \pi i x^{\prime} \nu_{a}\right) \mathrm{d} x^{\prime}, \\
= & \int \operatorname{rect}\left\{\frac{x^{\prime}}{2 \Delta x_{a}\left[1-\left|2\left(x_{a}-x_{a c}\right) / \Delta x_{a}\right|\right]}\right\} \\
& \times \exp \left(-2 \pi i x^{\prime} \nu_{a}\right) \mathrm{d} x^{\prime}, \\
= & 2 \Delta x_{a}\left[1-\left|\frac{2\left(x_{a}-x_{a c}\right)}{\Delta x_{a}}\right|\right] \\
& \times \operatorname{sinc}\left\{2 \Delta x_{a}\left[1-\left|\frac{2\left(x_{a}-x_{a c}\right)}{\Delta x_{a}}\right|\right] \nu_{a}\right\}
\end{aligned}
$$

for $\operatorname{rect}\left[\left(x_{a}-x_{a c}\right) / \Delta x_{a}\right]=1$ and $W_{\text {rect }}\left(x_{a}, \nu_{a}\right)=0$ for $\operatorname{rect}\left[\left(x_{a}-x_{a c}\right) / \Delta x_{a}\right]=0$. Observe that the Wigner distribution is nonzero only along the corridor defined by the rectangle function. This means that compaction in the ath domain to a certain interval will also result in compaction of the Wigner distribution to a corresponding corridor orthogonal to the $x_{a}$ axis.

Convolving $W_{\text {rect }}\left(x_{a}, \nu_{a}\right)$ with $W_{f}\left(x_{a}, \nu_{a}\right)$ in the $\nu_{a}$ direction will result in a broadening of $W_{f}\left(x_{a}, \nu_{a}\right)$ in the $\nu_{a}$ direction that is comparable with the width of $W_{\text {rect }}\left(x_{a}, \nu_{a}\right)$ in the $\nu_{a}$ direction. The envelope of $W_{\text {rect }}\left(x_{a}, \nu_{a}\right)$ as a function of $\nu_{a}$ for a given value of $x_{a}$ is simply $1 / \pi \nu_{a}$, which one can see is independent of $x_{a}$. The width of the main lobe of this distribution in the $\nu_{a}$ direction is $\sim 1 / \Delta x_{a}$.

Because of the abrupt transitions of the rectangle function, the spread of the Wigner distribution in the orthogonal domain is somewhat larger than fundamentally necessary. By using well-known windowing functions with smoother transitions, ${ }^{18}$ it is possible to reduce this spread to the fundamental minimum dictated by the space-frequency uncertainty relation. 


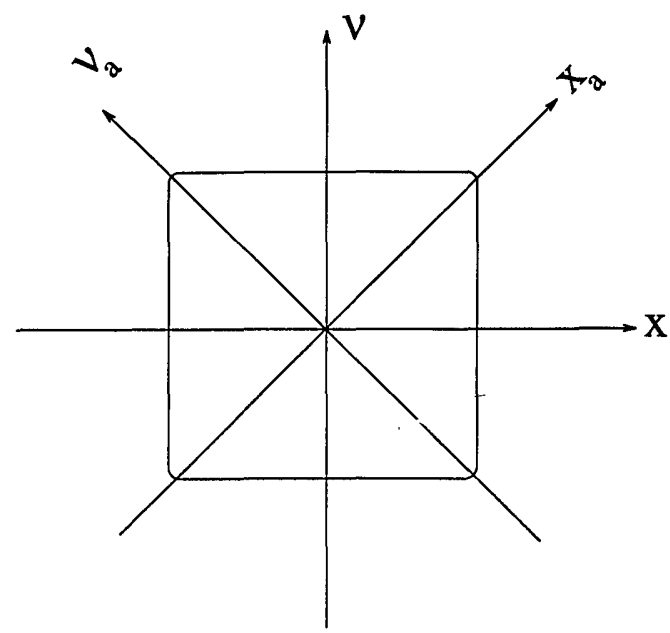

(a)

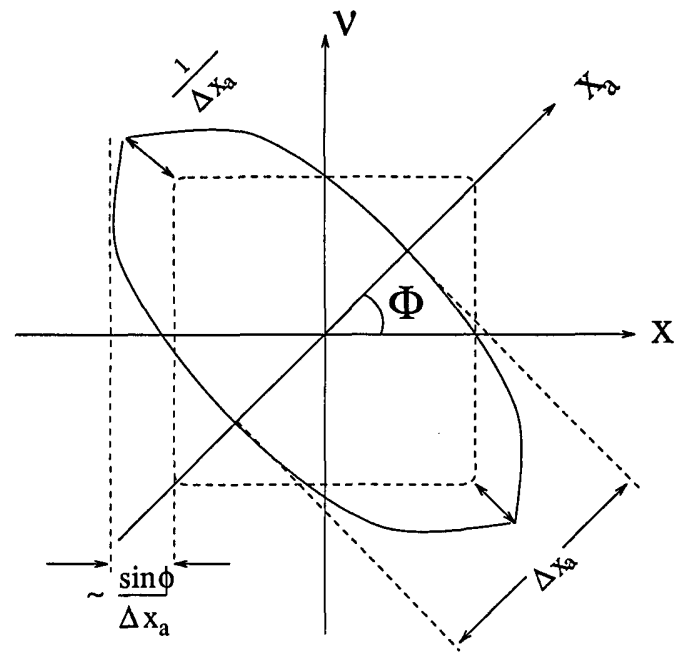

(b)

Fig. 2. (a) Wigner distribution of a signal, (b) compaction in the ath domain.

Thus we see that compaction in any domain down to an interval of width $\sim \Delta x_{a}$ necessarily results in a spread in the orthogonal domain by the amount $\sim 1 / \Delta x_{a}$. It also results in a spread $\propto|\sin \Delta \phi| / \Delta x_{a}$ in any other domain, where $\Delta \phi$ is the angle between the two domains (Fig. 2). Thus in general the uncertainty relation $\Delta x_{a} \Delta x_{a}{ }^{\prime} \geq$ $\sim\left|\sin \left(\phi-\phi^{\prime}\right)\right|$ holds, where $\Delta x_{a}$ is the spread of the representation in the ath domain. The exact value of the constant appearing on the right-hand sides of these uncertainty relations depends on the exact form of the functions involved.

It is important to note that this uncertainty is an inherent property of Wigner space. It is not meaningful to speak of regions in Wigner space with area smaller than $\Delta x_{a} \Delta \nu_{a} \sim 1$. In other words this is the smallest resolution of detail in Wigner space. In Sections 7-9 we discuss several illustrative examples in which the Wigner distribution of some function is indicated by a bounded curve in Wigner space, as shown in Fig. 2(a). Such drawings are meant to suggest that most of the energy of the signal in question lies within those boundaries. We must remember that the uncertainty relation actually limits how sharply these boundaries may be defined.

\section{FILTERING AND SEPARATION OF NOISE AND DISTORTION IN FRACTIONAL DOMAINS}

Having explained the effect of compaction and convolution in fractional domains, we consider their application to filtering and separation of undesired noise and distortion. Working in the conventional Fourier domain, one is limited to linear space-invariant operations, that is, those that can be expressed in the form of ordinary convolution (in the zeroth domain). One is led to inquire whether any advantage can be gained by filtering in fractional domains, because fractional convolution need not be space invariant.

Let us begin by examining two extreme cases. Filtering the function $f(x)$ with $h(x)$ in the $a=0$ th domain corresponds to ordinary convolution: $g=f * h$. The corresponding linear transform kernel is $h\left(x-x^{\prime}\right)$. Filtering in the $a=1$ st domain corresponds to multiplication $g=f h$. The corresponding kernel is $h\left(x^{\prime}\right) \delta\left(x-x^{\prime}\right)$.

It is instructive to illustrate these extremes with discrete signals. Any linear operation can be expressed as a matrix operator. The matrix operator corresponding to filtering in the $a=0$ th domain is Toeplitz, whereas that corresponding to filters in the $a=1$ st domain is diagonal. In any other given ath domain, the matrix will again have a special form other than these two extreme forms (so that it is still a restricted subset of all possible linear operations).

The output of our system $\mathbf{g}=\mathbf{f}$ 采 $\mathbf{h}$ satisfies

$$
\begin{aligned}
\mathbf{F}^{a} \mathbf{g} & =\mathbf{F}^{a} \mathbf{h} * \mathbf{F}^{a} \mathbf{f}, \\
\mathbf{F}^{a+1} \mathbf{g} & =\boldsymbol{\Lambda}\left[\mathbf{F}^{a+1} \mathbf{h}\right] \mathbf{F}^{a+1} \mathbf{f}, \\
\mathbf{g} & =\mathbf{F}^{-(a+1)} \boldsymbol{\Lambda}\left[\mathbf{F}^{a+1} \mathbf{h}\right] \mathbf{F}^{a+1} \mathbf{f} .
\end{aligned}
$$

Here $\boldsymbol{\Lambda}[\mathbf{y}]$ is a diagonal matrix with elements corresponding to the elements of the vector $\mathbf{y}$. Thus, if $\mathbf{T}$ denotes the linear transformation kernel such that $\mathbf{g}=\mathbf{T f}$, we have

$$
\mathbf{T}=\mathbf{F}^{-(a+1)} \boldsymbol{\Lambda}\left[\mathbf{F}^{a+1} \mathbf{h}\right] \mathbf{F}^{a+1}=\mathbf{F}^{-(a+1)} \boldsymbol{\Lambda}\left[\mathbf{F} \mathbf{h}_{a}\right] \mathbf{F}^{a+1},
$$

where $\mathbf{h}_{a}$ is the $a$ th transform of $\mathbf{h}$. The general kernel given above reduces to Toeplitz or diagonal form for $a=0$ and $a=-1$, respectively, as expected. Note that, if we are working with sequences of length $N$, $\mathbf{T}$ has only $N$ degrees of freedom. A further manipulation of the final equation brings out the form of $\mathbf{T}$ more clearly. It is known that, for any vector $\mathbf{y}$, the matrix $\mathbf{F}^{-1} \boldsymbol{\Lambda}[\mathbf{y}] \mathbf{F}^{1}$ is of Toeplitz form. Thus $\mathbf{T}$ is of the form $\mathbf{F}^{-a}\left[\right.$ Toeplitz] $\mathbf{F}^{a}$.

A general development of filtering in fractional domains would occupy a full-length paper itself and is not attempted here. Rather, we illustrate the usefulness of the fractional domain concept in visualization of the separation of noise or distortion from the desired signal. To provide motivation, we begin with an elementary example. Consider a signal plus distortion such that their conventional Fourier transforms do not overlap. Such distortion is easy to eliminate in Fourier space by use of a filter with a value of unity throughout the extent of the signal and a value of zero elsewhere. Such operations are particularly easy to implement in optical systems, because of the simplicity of the filter, which can be implemented as a binary amplitude mask. This is an example of a case in which the signal and distortion do not overlap in the $a=1$ st do- 


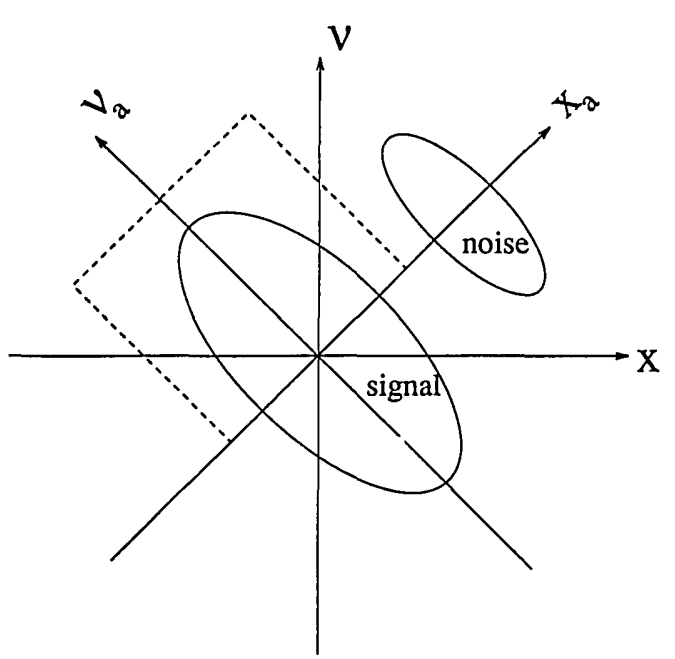

Fig. 3. Noise separation in the ath domain.

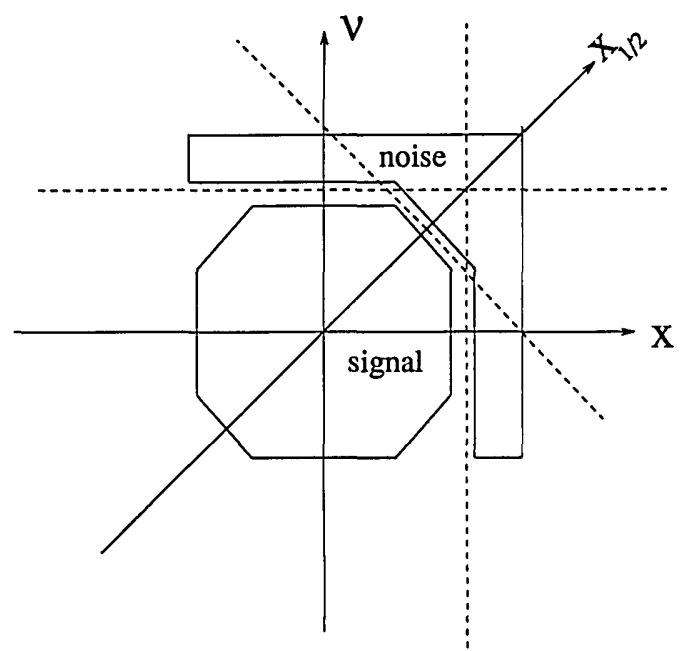

Fig. 4. Noise separation by repeated filtering in several fractional domains.

main, although they may overlap in the $a=0$ th domain. It is equally easy to separate signals that do not overlap in the $a=0$ th domain, although they may overlap in other domains.

Now consider Fig. 3, in which the Wigner distribution of a desired signal and undesired noise is shown. ${ }^{19}$ Considering the projections of these Wigner distributions on the $x$ and $\nu$ axes, we see that they overlap in both the $a=0$ th and $a=1$ st domains, although they do not overlap in Wigner space as a whole. It is not possible to mask away the undesired noise in either of the conventional domains. However, the noise is eliminated easily by the use of a simple binary amplitude mask in the ath domain, because the projections of their Wigner distributions do not overlap in this domain. It is worth stressing the ease of optical implementation of this operation. We can transform the signals to the ath domain by use of a piece of quadratic GRIN medium of length $a L$, then use a mask consisting of a piece of opaque material from which certain portions have been cut out, and finally transform back into the desired domain.

We now move on to another example, as illustrated in Fig. 4. Although the desired signal and noise do not overlap in Wigner space, their projections overlap in all do- mains. There is no domain in which a simple binary mask can separate the noise in a single step. However, separation of noise from signal can be accomplished by three consecutive filtering operations in the $a=0, a=$ $1 / 2$, and $a=1$ st domains. An optical implementation would involve first a mask in the space domain, followed by a GRIN medium of length $L / 2$, another mask in the $a=1 / 2$ th domain, another piece of GRIN medium of same length, a final mask in the $a=1$ st domain, and an inverse Fourier-transform operation. As can be seen, noise separation for space-variant signals and noise may involve the use of several filters in several domains.

Now we must take a closer look at the effect of using binary on-off amplitude mask filters. The use of such filters in the ath domain corresponds to compaction in that domain. Thus, when we separate the desired signal from noise in one domain, this leads to spread of the Wigner distribution in the orthogonal domain. If certain conditions are not met in a situation such as that illustrated by Fig. 4, this may result in merging of the Wigner distributions of signal and noise along that direction. Let us consider the simpler case of Fig. 5. Here we wish to cleanse a signal of space-bandwidth product $S B=$ $\Delta x \Delta \nu \gg 1$ from noise that is separated from the signal by the distances shown in Fig. 5. As we discussed in Section 6 , if we use a rectangular mask of the form $\operatorname{rect}(x / \Delta x)$ to eliminate the noise, this will result in broadening of the Wigner distribution of the signal by an amount of the order of $\sim 1 / \Delta x$ in the $\nu$ direction. Because we do not want the signal to mix with the noise in this process, we require that $\delta>1 / \Delta x=\Delta \nu / S B \ll \Delta \nu$. This is a reasonable requirement, being a direct consequence of the uncertainty relationship between space and frequency, as one can see by considering the following interpretation. Notice we are merely requiring that the value of the hatched area in Fig. 5 satisfy $\delta \Delta x>1$. This is the smallest meaningful area in Wigner space; we cannot hope to work in Wigner space with greater precision, and thus a buffer region of this area must separate the signal and the noise if we are to hope to separate them by any method.

Let us also point out how our considerations relate to Wiener filtering. We considered examples in which the

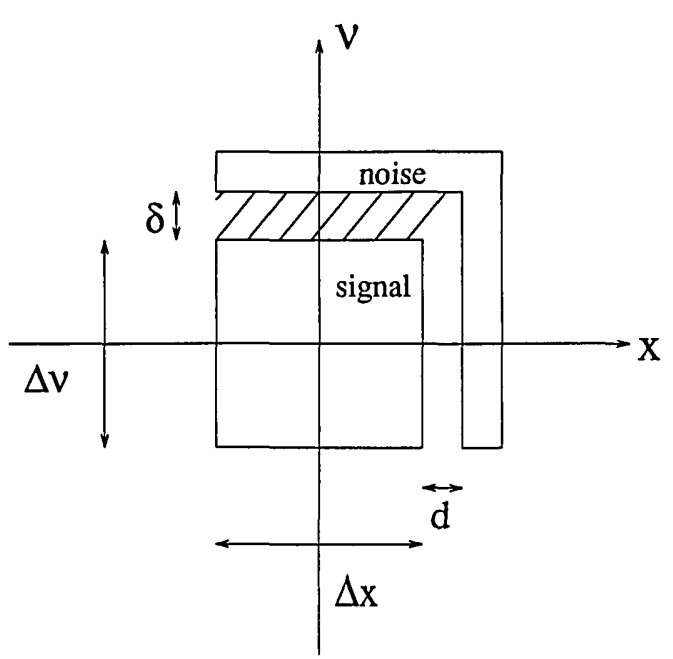

Fig. 5. Limits to noise separation imposed by the uncertainty relation. 
desired signal and noise do not have significant overlap in Wigner space and are sufficiently separated. In this case near-perfect recovery of the signal is possible. The theory of Wiener filtering is in fact more general and provides the optimal filter, minimizing mean-square error even when the Wigner distributions of signal and noise overlap and perfect recovery is not possible. Although we do not consider such examples because they do not lend themselves to easy graphic interpretation and add a degree of complexity that obscures the main point of the present discussion, the generalization of least-mean-square error filtering to fractional domains should be possible.

Ultimately it is important to note that filtering in a fractional domain, or several fractional domains in cascade, is still a linear operation whose overall effect can be captured in a kernel of the form $h\left(x, x^{\prime}\right)$. The optimal kernel (in the least-mean-square error sense) can be found by other means, without the introduction of the concept of fractional Fourier transforms. However, viewing these operations as filtering in fractional domains is a useful interpretation that adds transparency and meaning to the operation being done. More important, this interpretation permits easy implementation in optical systems. The implementation of general space-variant linear transformations in optics with conventional methods such as matrix multipliers is notoriously wasteful of the spacebandwidth product. $^{20}$ In practice it is impossible to realize such operations effectively for large two-dimensional images, because the space-bandwidth product needed is the square of the number of pixels in the image. However, as illustrated by our examples, the fractional domain interpretation leads to simple and efficient implementations. We do not know whether the same advantage holds in digital signal processing or whether direct matrix multiplication methods would be preferred.

Let us briefly revisit the example illustrated in Fig. 4 so as to provide motivation for Section 8. For the sake of notational convenience, using our notation for discrete signals, we may write the overall operation as

$$
\mathbf{g}=\mathbf{F}^{3} \Lambda\left[\mathbf{h}_{3}\right] \mathbf{F}^{1 / 2} \Lambda\left[\mathbf{h}_{2}\right] \mathbf{F}^{1 / 2} \Lambda\left[\mathbf{h}_{1}\right] \mathbf{f},
$$

where we remember that $\mathbf{F}^{3}=\mathbf{F}^{-1}$. The $\mathbf{h}_{j}$ are simply multiplicative filter sequences that, in our example, would have either unity or zero as the values of their components.

\section{GENERALIZED SPATIAL FILTERING}

Generalizing Eq. (68), we can write

$$
\begin{aligned}
\mathbf{g} & =\mathbf{T f}, \\
\mathbf{T} & =\mathbf{F}^{a_{M}} \Lambda\left[\mathbf{h}_{M}\right] \cdots \Lambda\left[\mathbf{h}_{3}\right] \mathbf{F}^{a_{2}} \Lambda\left[\mathbf{h}_{2}\right] \mathbf{F}^{a_{1}} \Lambda\left[\mathbf{h}_{1}\right] \mathbf{F}^{a_{0}} .
\end{aligned}
$$

Such a system is realized by cascading a piece of GRIN medium of length $a_{0} L$ (which performs $\mathbf{F}^{a_{0}}$ ) with a mask of transmittance $\mathbf{h}_{1}$, followed by a piece of GRIN medium of length $a_{1} L$, and so on. By choosing the number of filters $M$ and the masks $\mathbf{h}_{j}$ appropriately, it should be possible to realize any desired general linear transformation T. This idea has been discussed at length elsewhere. ${ }^{3}$ The reader is referred to this reference for details; however, the problem of how to choose the masks for given $\mathbf{T}$ is still open.

Here we satisfy ourselves by noting a special case of
Eq. (70). If we take $a_{0}, a_{1}, \ldots$ all equal to unity, we obtain

$$
\mathbf{T}=\mathbf{F} \boldsymbol{\Lambda}\left[\mathbf{h}_{M}\right] \cdots \boldsymbol{\Lambda}\left[\mathbf{h}_{3}\right] \mathbf{F} \boldsymbol{\Lambda}\left[\mathbf{h}_{2}\right] \mathbf{F} \boldsymbol{\Lambda}\left[\mathbf{h}_{1}\right] \mathbf{F} .
$$

If we limit ourselves to even functions for simplicity, this corresponds to first multiplying the input function with a certain function, then convolving it with another function, then multiplying it, then convolving it, and so on.

\section{MULTIPLEXING IN FRACTIONAL DOMAINS}

We now discuss the concept of multiplexing in fractional domains. First, let us review what it means to multiplex in the space domain (or in the time domain) and in the frequency domain. Multiplexing in the space domain involves packing together signals whose representations are compact in this domain. We simply shift the several functional representations in this domain with respect to one another so that they do not overlap and are easily separated later on. [If the original signals are not compact (or are too wide to be practically considered compact), they can be cut into many smaller compact pieces, which are then compressed spatially and interleaved with other signals.]

On the other hand, multiplexing in the frequency domain involves packing together signals that are compact in the frequency domain. Again, we simply shift the several functional representations in this domain with respect to one another so that they do not overlap and are easily separated later on. (This may be accomplished by modulation in the space domain.)

It is instructive to view these processes in Wigner space. Let the total extent of the aperture of our system be $\Delta x_{\text {total }}$ and the double-sided spatial bandwidth of our system be $\Delta \nu_{\text {total }}$. (Alternatively, we may represent the total transmission time as $\Delta t_{\text {total }}$ and the bandwidth of the transmission medium as $\Delta f_{\text {total }}$ for temporal systems.) This defines a region in Wigner space that we are free to use in packing signals. First, let us consider that the extents of our signals $\Delta x$ are approximately equal to $\Delta x_{\text {total }}$ but that their bandwidth $\Delta \nu$ is smaller than $\Delta \nu_{\text {total }}$ (Fig. 6). Then,

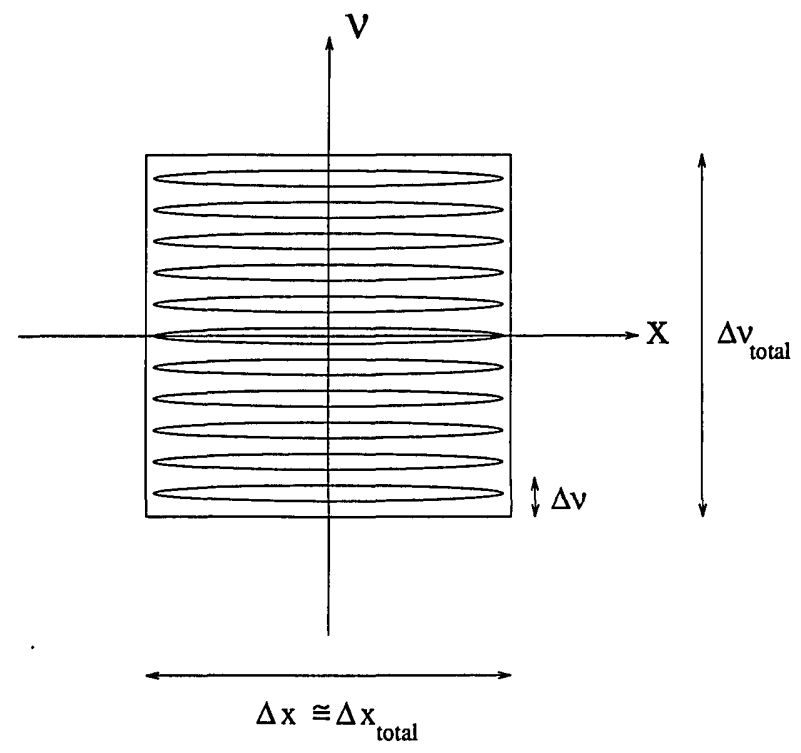

Fig. 6. Multiplexing in the frequency domain. 


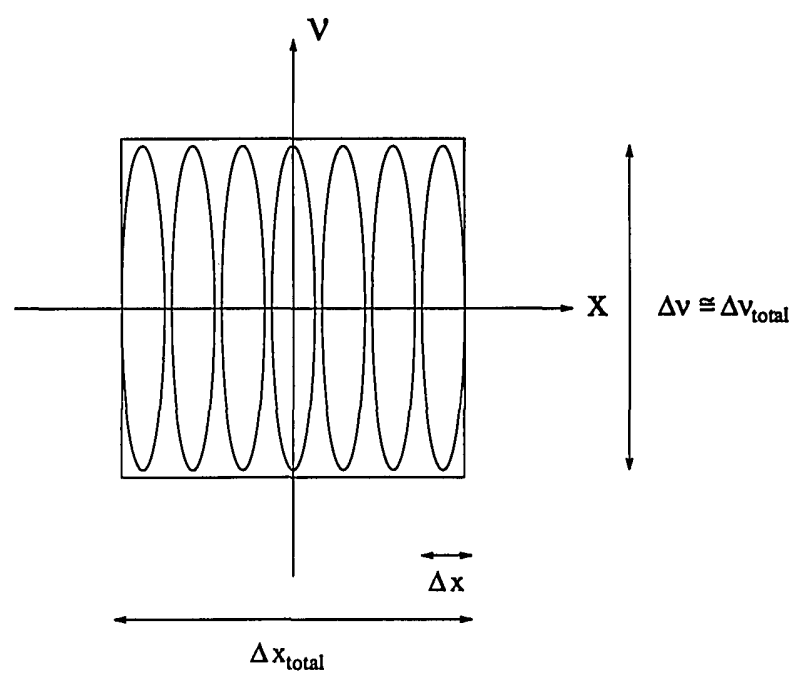

Fig. 7. Multiplexing in the space domain.

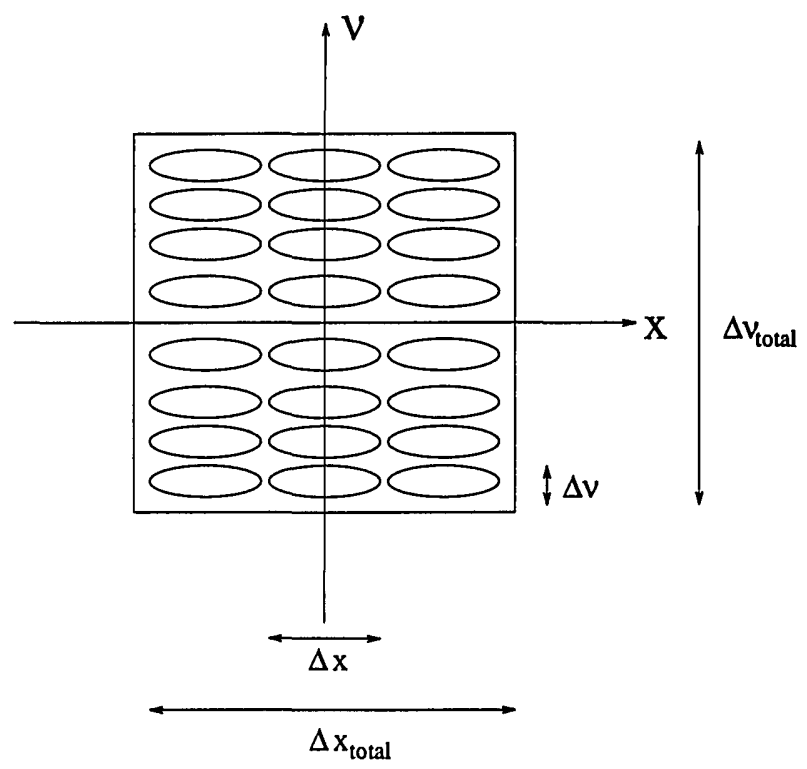

Fig. 8. Multiplexing in both space and frequency.

it is natural to pack several of these signals by using frequency domain multiplexing, as shown in Fig. 6. Contrarily, let us assume that the bandwidth of our signals is equal to $\Delta \nu_{\text {total }}$ but that the spatial extent is smaller than the overall spatial extent of our system. Then, it is natural to use space division multiplexing (Fig. 7).

If both $\Delta x$ and $\Delta \nu$ are smaller than $\Delta x_{\text {total }}$ and $\Delta \nu_{\text {total }}$, respectively, then both space and frequency domain multiplexing may be used together, as in Fig. 8. This is accomplished by shifting the Wigner distributions of the signals to be multiplexed by the appropriate amounts in space (by convolving them with delta functions) and frequency (by multiplying them with harmonics).

Now let us consider the oblique Wigner distribution illustrated in Fig. 9. It is evident that using the scheme of Fig. 8 is not the most efficient way of multiplexing such signals. The scheme illustrated in Fig. 10 is much more efficient. To pack signals in this manner, all we have to do is to transform them into the appropriate ath domain. In this domain, multiplexing these signals is accomplished in the same way as it was accomplished in Fig. 8.
It is easy to generalize this procedure to packing of several signals whose Wigner distributions are not identical. All we need to do is to shift the Wigner distributions of the signals in the appropriate directions in Wigner space so as to achieve the most efficient packing possible. Shifting in the $x$ direction involves convolution with a delta function. Shifting in the $\nu$ direction involves multiplication with a harmonic. Shifting in other directions involves convolution in the ath domain.

Of course, shifting the Wigner distribution of a signal in a direction along the $x_{a}$ axis can also be realized in two steps without going to the ath domain. We can first shift the signal in the $x=x_{0}$ direction by convolving it with a delta function and then shift it in the $\nu=x_{1}$ direction by multiplying it with a harmonic (or shift the signal in reverse order):

$$
\begin{aligned}
{\left[f_{0}\left(x_{0}\right) * \delta\left(x_{0}-x_{0 c}\right)\right] \exp \left(2 \pi i \nu_{0 c} x_{0}\right) } & \\
= & f\left(x_{0}-x_{0 c}\right) \exp \left(2 \pi i \nu_{0 c} x_{0}\right) .
\end{aligned}
$$

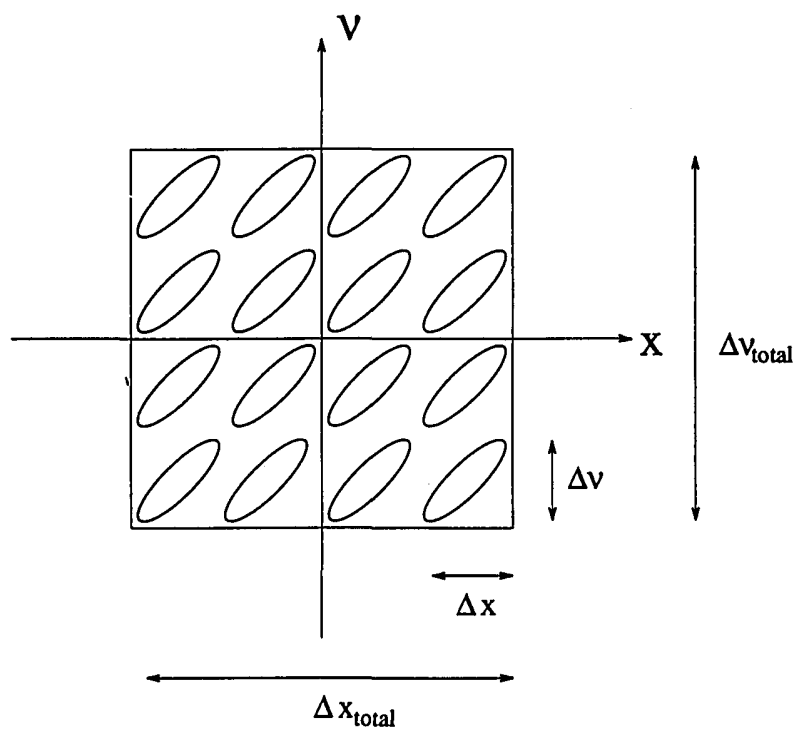

Fig. 9. Inefficient multiplexing of a signal with an oblique Wigner distribution.

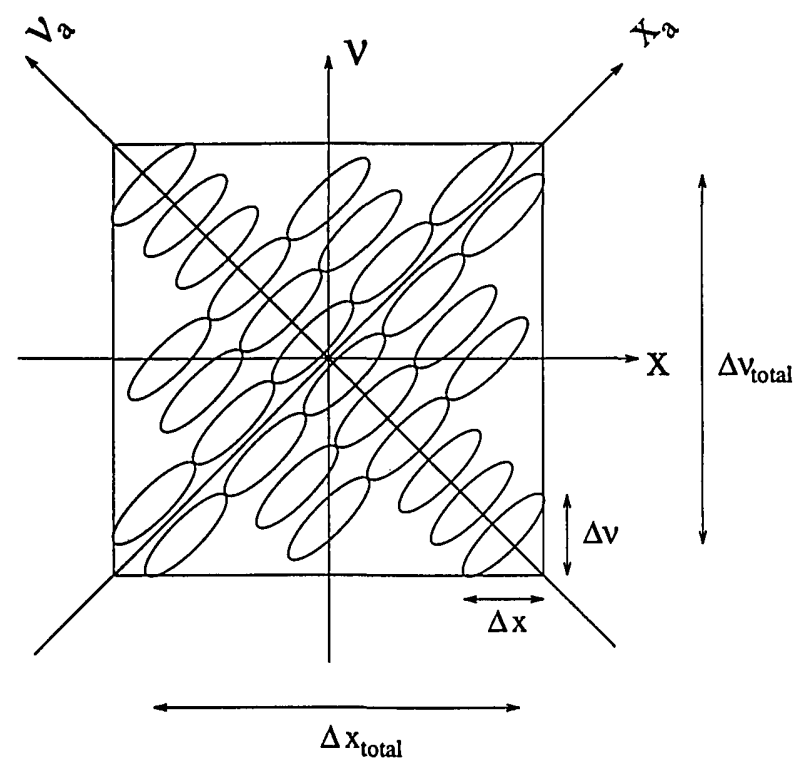

Fig. 10. Efficient multiplexing of a signal with an oblique Wigner distribution. 
The ath fractional transforms of Eq. (72) will be equivalent to shifting the function in the ath domain:

$$
f_{a}\left(x_{a}\right) * \delta\left(x_{a}-x_{a c}\right)=f_{a}\left(x_{a}-x_{a c}\right),
$$

within phase factors, which will not appear in their Wigner distributions.

An additional consideration in multiplexing would be taking into account the Wigner distribution of any noise and distorting signals and avoiding those regions of Wigner space where they are concentrated.

\section{EXTENSIONS}

In specific applications it might be of interest to find the choice of $x$ and $\nu$ axes that are most natural and physically meaningful. These might not always be self-evident, just as the normal coordinates of a physical system are not always the ones that we are initially inclined to assign.

It also seems to be of interest to analyze fractional Hankel transforms. These would be of interest for optical systems in which there exists circular symmetry but in which noise and distortion may have a radial dependence. If a Wigner space in which one dimension is radial distance and the other is radial frequency is defined, such undesired noise would have an energy density whose distribution in frequency depends on the radial variable, so that filtering methods similar to those discussed in this paper may be of interest.

As mentioned above, the generalization to two-dimensional and temporal systems is relatively easy from a mathematical viewpoint; however, different and interesting interpretations may be involved in the study of such systems. Further study of discrete systems is also of interest.

Finally, we note that the algebra of fractional Fourier transforms is far from complete, and we expect several new, at present unforeseen identities and results to be discovered in the future. As one example, the function of polar coordinates $(r, \phi)$ defined by $\left|\mathscr{F}^{2 \phi / \pi}[f](r)\right|^{2}$ was seen to be equal to the Radon transform of the Wigner distribution of $f(x)$. The interpretation of $\mathscr{F}^{2 \phi / \pi}[f](r)$, however, is not known.

\section{CONCLUSION}

What we know as the space and spatial frequency domains are merely special cases of fractional domains. These domains are characterized by the parameter $a$. The representation of a signal in the $a$ th domain is the ath fractional Fourier transform of its representation in the $a=0$ th domain, which we define to be the space domain. The representation in the $a=1$ st domain is the conventional Fourier transform. If we set up a two-dimensional space, called the Wigner space, such that one axis $(x)$ corresponds to the $a=0$ th domain (the conventional space domain) and the other ( $\nu$ ) corresponds to the $a=1$ st domain (the conventional spatial frequency domain), then the ath domain corresponds to an axis making an angle $\phi=a \pi / 2$ with the $x$ axis. One can alternatively obtain the representation of a signal in the ath domain (without phase information) by taking the projection of the energy distribution of the signal in this space (its Wigner distribution) on this axis.
The energy distribution of a signal in Wigner space should be considered a geometric entity, independently of the choice of the space and frequency axes. The introduction of these axes merely corresponds to choosing arbitrarily the origin of the parameter $a$. We speak of the $a=$ 0 th domain as the space domain, the $a=1$ st domain as the frequency domain, and so on. Other fractional domains are in no way inferior to those domains and have the same properties.

Fractional Fourier transforms are inherently related to chirp transforms, because chirp functions are nothing but the $a=0$ domain representation of signals that appear as either delta functions or harmonics in other fractional domains. Fractional Fourier transforms are also related to wavelet transforms. The wavelet transform of a onedimensional function has two parameters instead of one. Likewise, the fractional Fourier transform $f_{a}\left(x_{a}\right)$ of the function $f(x)$ has two parameters, $x$ and $a$. It is possible to establish a relationship between the two transforms by choosing a chirp function as the wavelet transform kernel.

A desired signal and noise may overlap in both conventional space and frequency domains but not in a particular fractional domain. Even when this is not the case, binary amplitude masking in a few fractional domains in cascade may enable one to eliminate noise quite conveniently. The optical implementation of a system for this purpose consists of several masks sandwiched between segments of quadratic GRIN medium.

Multiplexing in fractional domains can offer greater efficiency in space-bandwidth product use than conventional space division or frequency division multiplexing, if the Wigner distribution of the signals is aligned better with a fractional domain rather than with one of the conventional domains.

Ultimately, what is most remarkable from an optics viewpoint is the ease with which the two-dimensional fractional Fourier transform can be realized. Thus, as the signal processing community generalizes its operations to fractional domains, the optics community will be able to generalize to a fractional Fourier optics.

\section{APPENDIX A: EIGENFUNCTIONS OF THE FOURIER TRANSFORM OPERATOR}

It is actually quite easy to obtain eigenfunctions (also known as self-Fourier functions) of the Fourier-transform operator. If $g(x)$ is a transformable function with Fourier transform $G(\nu)$, then $f(x)=g(x)+G(x)+g(-x)+$ $G(-x)$ is an eigenfunction. ${ }^{21}$ It was also shown that any eigenfunction can be decomposed in this manner. ${ }^{22}$

We saw that any (well-behaved) function can be expanded into the Hermite-Gauss function set; i.e., this set is complete. It was shown that the expansion over the members of this set [Eq. (7)] can be grouped into four partial sums according to their eigenvalues. ${ }^{10}$ An alternative and shorter derivation of this result by Lohmann ${ }^{23}$ is given below.

Consider a function $f(x)$ and construct the functions

$$
4 f_{j}(x)=f(x)+i^{j} F(x)+i^{2 j} f(-x)+i^{3 j} F(-x)
$$

for $j=0,1,2,3$. It is easy to verify that each of these functions is an eigenfunction of the Fourier-transform 
operator, with eigenvalues $1,-i,-1, i$ for $j=0,1,2,3$, respectively. Furthermore, it is also easy to see that

$$
f(x)=\sum_{j=0}^{3} f_{j}(x)
$$

which proves the desired result.

Further results on eigenfunctions of the fractional Fourier operator may be found in Ref. 24 .

\section{APPENDIX B: FRACTIONAL FOURIER-TRANSFORMING PROPERTY OF QUADRATIC GRIN MEDIA}

The fractional Fourier-transforming property of quadratic GRIN media was shown in Refs. 1-3. Here we provide a much shorter and transparent proof.

The refractive-index distribution of quadratic GRIN media is given by ${ }^{25}$

$$
n^{2}(r)=n_{1}^{2}\left[1-\left(n_{2} / n_{1}\right) r^{2}\right],
$$

where $r$ is the radial distance from the optical $z$ axis and $n_{1}, n_{2}$ are physical parameters of the medium. If a light distribution of the form $f(x, y)$ is incident from the left at $z=0$, at $z=a L \equiv a(\pi / 2)\left(n_{1} / n_{2}\right)^{1 / 2}$ we observe $F^{a}[f(x, y)]$, the ath Fourier transform of $f(x, y)$. Thus a piece of quadratic GRIN medium of length $a L$ acts as an ath fractional Fourier transformer. We now derive this result.

Propagation in GRIN media is governed by an equation identical in form to Eq. (3). Thus the eigenmodes of propagation are also Hermite-Gauss functions as in Eq. (5) but scaled with the appropriate physical parameters. $^{2}$ [Substituting unity for the value of the physical scale parameter $s$ (dimension length) in Ref. 2 gives Eq. (5).]

To analyze the effect of propagation over a distance $\Delta z$ on an input function $f(x)$, we may again express it in terms of the eigenmodes of propagation as in Eqs. (7) and (8). Then, the output $g(x)$ is given by ${ }^{2}$

$$
g(x)=\sum_{n=0}^{\infty} \tilde{A}_{n} \lambda_{n}{ }^{\prime}(\Delta z) \tilde{\Psi}_{n}(x),
$$

where $\lambda_{n}{ }^{\prime}(\Delta z)=\exp \left(i \beta_{n} \Delta z\right)$ are the eigenvalues associated with propagation over a distance $\Delta z$ in such a medium (as shown in Ref. 25) and

$$
\beta_{n} \simeq k-\left(n_{2} / n_{1}\right)^{1 / 2}(n+1 / 2)
$$

is the propagation constant for the $n$th mode and $k$ is the propagation constant in a homogeneous medium of refractive index $n_{1}$.

Let us choose $\Delta z=a L \equiv a(\pi / 2)\left(n_{1} / n_{2}\right)^{1 / 2}$. On substitution we find that

$$
\begin{aligned}
\lambda_{n}{ }^{\prime}(a L) & =\exp (i k a L-i a \pi / 4) \exp (-i a n \pi / 2)=C i^{-a n} \\
& =C\left(i^{-n}\right)^{a}=C{\lambda_{n}}^{a},
\end{aligned}
$$

where $C$ is a constant associated with the optical phase retardation $k a L-a \pi / 4$ and has no effect on the transverse functional form of the output, because it does not depend on $n$ and can be taken out of the summation. Apart from this inconsequential factor, we see that the eigenvalues for propagation over a distance $a L$ are the same as the eigenvalues of the ath fractional Fourier- transform operation [Eqs. (10) and (11)]. This means that the operations represented in Eqs. (11) and (B2) are equivalent. Thus propagation over a distance $a L$ in a quadratic GRIN medium results in the ath fractional Fourier transformation. This is the result we sought to prove.

By using the propagation expressions given in Refs. 1 and 2, if a light distribution of the form $f(y / s)$ (with $y$ given in meters) is incident upon one end of a quadratic GRIN medium, after propagation over a length $a L$, we observe the light distribution

$$
\exp [i(k a L-a \pi / 4)] f_{a}(y / s),
$$

where $f_{a}(x)$ is the ath fractional transform of $f(x)$. Here, $s$ is a parameter with dimensions of length and is related to the GRIN medium parameters as $s=$ (wavelength $^{2} /$ $\left.n_{1} n_{2}\right)^{1 / 4}$ [ $s$ is analogous to the parameter (wavelength $\times$ focal length) appearing in classical Fourier optics].

Likewise the physical propagation kernel $B_{a}{ }^{\prime}\left(y, y^{\prime}\right)$ is related to the mathematical propagation kernel $B_{a}\left(x, x^{\prime}\right)$ as

$$
B_{a}{ }^{\prime}\left(y, y^{\prime}\right)=\exp [i(k a L-a \pi / 4)] s^{-1} B_{a}\left(y / s, y^{\prime} / s\right) .
$$

Once again the reader is referred to Refs. 1-3 for twodimensional versions of these results.

\section{APPENDIX C: EFFECT OF THE FRACTIONAL FOURIER TRANSFORM ON THE WIGNER DISTRIBUTION FUNCTION}

Here we show that the Wigner distribution of the $a$ th fractional Fourier transform of $f(x)$ is the same as the Wigner distribution of $f(x)$ rotated by $\phi=a \pi / 2$ [Eq. (16)]. Because the derivation is straightforward yet lengthy, we merely sketch the steps, leaving it to the reader to fill in the details.

Equation (12) gives $\left(\mathscr{F}^{a}[f]\right)(x)$ in the form of a linear transformation, with $B_{a}(x, v)$ being given by Eq. (14):

$$
f_{a}(x)=\left(F^{a}[f]\right)(x)=\int B_{a}(x, v) f(v) \mathrm{d} v .
$$

The Wigner distribution of $\mathscr{F}^{a}[f]$ can be evaluated by substitution of this integral expression in the definition of the Wigner distribution [Eq. (15)], resulting in an expression involving three integrals over $v, v^{\prime}, x^{\prime}$ (if we let the dummy variable appearing in the second occurence of $\mathscr{F}^{a}[f]$ be $v^{\prime}$ ). After some manipulation, we are faced with an integral of the form

$$
\int \exp \left\{-2 \pi i\left[\nu-x \cot \phi+\left(v+v^{\prime}\right) / 2 \sin \phi\right] x^{\prime}\right\} \mathrm{d} x^{\prime}
$$

inside the expression with which we are working. This is equal to $\delta\left[\nu-x \cot \phi+\left(v+v^{\prime}\right) / 2 \sin \phi\right]$. This delta function will eliminate the integral over $v^{\prime}$ by virtue of the sifting property, leaving us with

$$
\begin{aligned}
\exp [-4 \pi i(\nu & \sin \phi-x \cos \phi) x / \sin \phi] \\
& \times \exp \left[-4 \pi i(\nu \sin \phi-x \cos \phi)^{2}\right] \cot \phi \\
& \times \int \exp [-4 \pi i v(x \sin \phi+\nu \cos \phi)] \\
& \times f(v) f^{*}[-v-2(\nu \sin \phi-x \cos \phi)] \mathrm{d} v .
\end{aligned}
$$

Now we wish to show that this is the Wigner distribution of $f(x)$ rotated by $\phi$. If we use the rotational 
transformation,

$$
\begin{aligned}
x^{\prime} & =x \cos \phi-\nu \sin \phi, \\
\nu^{\prime} & =x \sin \phi+\nu \cos \phi,
\end{aligned}
$$

and subsequently the change of integration variable $v=$ $v^{\prime} / 2+x^{\prime}$, the expression at hand reduces to Eq. (15), the definition of the Wigner transformation of $f(x)$. This is the result that we sought to prove.

\section{APPENDIX D: EIGENVALUES AND EIGENFUNCTIONS OF THE DISCRETE FRACTIONAL FOURIER-TRANSFORM OPERATOR}

Let us apply the operator $\mathbf{F}^{a}$ on the eigenvector $\mathbf{g}_{n}$ of $\mathbf{F}$ with eigenvalue $\lambda_{n}$. On substitution of Eq. (34) into Eq. (33),

$$
\mathbf{F}^{a} \mathbf{g}_{n}=\sum_{j=0}^{3} \sum_{k=1}^{4} \frac{1}{4} \exp [i k(a-j) \pi / 2] \mathbf{F}^{j} \mathbf{g}_{n} .
$$

Using Eqs. (30) and (32) and rearranging, we obtain

$$
\mathbf{F}^{a} \mathbf{g}_{n}=\sum_{k=1}^{4} \exp (i k a \pi / 2) \frac{1}{4} \sum_{j=0}^{3} \exp [i j(-n-k) \pi / 2] \mathbf{g}_{n} \text {. }
$$

The inner summation is simply $\delta_{-n, k}$, by virtue of Eq. (28), so that we end up with

$\mathbf{F}^{a} \mathbf{g}_{n}=\sum_{k=1}^{4} \exp (i k a \pi / 2) \delta_{-n, k} \mathbf{g}_{n}=\exp (-i n a \pi) /{ }^{2} \mathbf{g}_{n}=\lambda_{\mathrm{n}}^{\mathrm{a}} \mathbf{g}_{n}$,

which is the desired result, Eq. (35).

\section{ACKNOWLEDGMENTS}

It is a pleasure to acknowledge the contribution of Adolf W. Lohmann of the University of Erlangen-Nürnberg, which were made in the form of many discussions and suggestions. He has been a constant source of inspiration throughout this research. The concept of multiplexing in fractional domains was first suggested to us by Bülent Sankur of Boğaziçi University, Istanbul, Turkey. We also thank Enis Çetin of Bilkent University for several discussions and suggestions.

Note added in proof: The following papers, which discuss related concepts, have recently been brought to our attention: N. G. De Bruijn, "A theory of generalized functions with applications to Wigner distribution and Weyl correspondence," Nieuw Arch. Wiskunde (3) XXI, 205280 (1973); D. Mihovilovic and R. N. Bracewell, "Adaptive chirplet representation of signals on time-frequency plane," Electron. Lett. 27, 1159-1161 (1991); J. Wood and D. T. Barry, "Radon transformation of the Wigner spectrum," in Advanced Signal Processing Algorithms, Architectures, and Implementations III, F. T. Luk, ed., Proc. Soc. Photo-Opt. Instrum. Eng. 1770, 358-375 (1992); L. B. Almeida, "An introduction to the angular Fourier transform," in Proceedings of IEEE International Conference on Acoustics, Speech, and Signal Processing (Institute of Electrical and Electronics Engineers, New York, 1993), pp. III-257-III-260 (1993).

\section{REFERENCES AND NOTES}

1. H. M. Ozaktas and D. Mendlovic, "Fourier transforms of fractional order and their optical interpretation," Opt. Commun. 101, 163-169 (1993).

2. D. Mendlovic and H. M. Ozaktas, "Fractional Fourier transformations and their optical implementation: I," J. Opt. Soc. Am. A 10, 1875-1881 (1993).

3. H. M. Ozaktas and D. Mendlovic, "Fractional Fourier transformations and their optical implementation: II," J. Opt. Soc. Am. A (to be published).

4. A. C. MćBride and F. H. Kerr, "On Namias's fractional Fourier transform," IMA J. Appl. Math. 39, 159-175 (1987).

5. V. Namias, "The fractional Fourier transform and its application in quantum mechanics," J. Inst. Math. Its Appl. 25, 241265 (1980).

6. D. Mendlovic, H. M. Ozaktas, and A. W. Lohmann, "Fourier transforms of fractional order and their optical interpretation," in Optical Computing, Vol. 7 of 1993 OSA Technical Digest Series (Optical Society of America, Washington, D.C., 1993), pp. 127-130.

7. A. W. Lohmann, "Image rotation, Wigner rotation, and the fractional Fourier transform," J. Opt. Soc. Am. 10, 21812186 (1993).

8. D. Mendlovic, H. M. Ozaktas, and A. W. Lohmann, "The effect of propagation in graded index media on the Wigner distribution function and the equivalence of two definitions of the fractional Fourier transform," Appl. Opt. (to be published).

9. N. Wiener, The Fourier Integral and Certain of Its Applications (Cambridge U. Press, Cambridge, 1933).

10. G. Cincotti, F. Gori, and M. Santarsiero, "Generalized selfFourier functions," J. Phys. A 25, 1191-1194 (1992).

11. H. O. Bartelt, K.-H. Brenner, and A. W. Lohmann, "The Wigner distribution function and its optical production," Opt. Commun. 32, 32-38 (1980).

12. T. A. C. M. Claasen and W. F. G. Mecklenbraucker, "The Wigner distribution-a tool for time-frequency signal analysis; part 1: continuous-time signals," Philips J. Res. 35, 217-250 (1980).

13. T. A. C. M. Claasen, and W. F. G. Mecklenbraucker, "The Wigner distribution-a tool for time-frequency signal analysis; part 2: discrete-time signals," Philips J. Res. 35, 276300 (1980).

14. A. W. Lohmann and B. H. Soffer, "Relationship between two transforms: Radon-Wigner and fractional Fourier," in $\mathrm{An}$ nual Meeting, Vol. 16 of 1993 OSA Technical Digest Series (Optical Society of America, Washington, D.C., 1993), p. 109.

15. A. Rosenfeld and A. C. Kak, Digital Picture Processing, 2nd ed. (Academic, San Diego, Calif., 1982), Vol. 1.

16. B. W. Dickinson and K. Steiglitz, "Eigenvectors and functions of the discrete Fourier transform," IEEE Trans. Acoust. Speech Signal Process. ASSP-30, 25-31 (1982).

17. L. Onural, "Diffraction from a wavelet point of view," Opt. Lett. 18, 846-848 (1993).

18. A. V. Oppenheim and R. W. Shafer, Digital Signal Processing (Prentice-Hall, Englewood Cliffs, N.J., 1975).

19. Our somewhat artificial examples have been chosen to illustrate the essential concepts in the simplest possible terms. In particular, it should be noted that real functions always have Wigner distributions exhibiting even symmetry with respect to $\nu$.

20. D. Mendlovic and H. M. Ozaktas, "Optical-coordinate transformation methods and optical interconnection architectures," Appl. Opt. 32, 5119-5124 (1993).

21. M. J. Caola, "Self-Fourier functions," J. Phys. A 24, 11431144 (1991).

22. A. W. Lohmann and D. Mendlovic, "Self-Fourier objects and other self-transform objects," J. Opt. Soc. Am. A 9, 20092012 (1992).

23. A. W. Lohmann, University of Erlangen-Nürnberg, Staudtstrasse 7, Erlangen, Germany (personal communication).

24. D. Mendlovic, H. M. Ozaktas, and A. W. Lohmann, "Self Fourier functions and fractional Fourier transforms," Opt. Commun. (to be published).

25. A. Yariv, Optical Electronics, 3rd ed. (Holt, New York, 1985) 